\title{
EHMTI-0169. Trajectories of headache days over one year (5 waves) in chronic and episodic migraineurs participating in the chronic migraine epidemiology and outcomes (cameo) study
}

\author{
RB Lipton ${ }^{1 *}$, D Serrano ${ }^{2}$, AM Adams³ ${ }^{3}$ DC Buse ${ }^{1}$, Al Scher $^{4}$ \\ From 4th European Headache and Migraine Trust International Congress: EHMTIC 2014 \\ Copenhagen, Denmark. 18-21 September 2014
}

\section{Introduction}

Within-person variation in headache (HA) frequency and the nature and predictors of this variation have rarely been studied.

\section{Aims}

To determine variation and predictors of HA days/month in persons with episodic (EM) or chronic migraine (CM).

\section{Methods}

CaMEO was a longitudinal US survey employing quota sampling for screening 80,783 respondents to identify persons with migraine. Baseline HA day frequency was classified as EM (modified ICHD-3b migraine diagnosis, $<15$ HA days/month for past 3 months) or CM (modified ICHD-3b diagnosis, $\geq 15$ HA days/month for past 3 months). Migraineurs completed quarterly surveys over 5 waves ( $\sim 1$ year) assessing HA symptoms and frequency. Using repeated measures regression modeling, we describe variation in HA days within/between individuals, and evaluate potential variation predictors.

\section{Results}

From 58,418 useable returns, we identified 16,789 migraineurs at baseline (EM: 15,313 [91.2\%], CM: 1,476 [8.8\%]). The reported number of HA days/month showed cyclic variation over time within and between respondents with both CM and EM. HA days increased more for CM (vs EM) persons over time (quarterly $\mathrm{RR}=1.26,95 \% \mathrm{CI}$
1.20-1.33; $\mathrm{P}<0.0001)$. A comprehensive graphical decomposition of this interaction will be presented.

\section{Conclusion}

Substantial within- and between-person heterogeneity in quarterly estimates of HA days/month was observed, and resulting trajectories differed between groups. The number of HA days/month increased $26 \%$ more per quarter for persons with CM vs EM, possibly resulting from a lowered threshold for migraine initiation, creating positive feedback leading to more attacks.

\section{Funding}

Allergan

\section{Authors' details}

${ }^{1}$ The Saul R. Korey Department of Neurology, Albert Einstein College of Medicine and Montefiore Headache Center, Bronx, USA. Biostatistics, Vedanta Research, Chapel Hill, USA. ${ }^{3}$ Global Medical Affairs, Allergan Inc., Irvine, USA. ${ }^{4}$ Department of Preventative Medicine and Biometrics, Uniformed Services University of the Health Sciences, Bethesda, USA.

Published: 18 September 2014

doi:10.1186/1129-2377-15-S1-D4

Cite this article as: Lipton et al:: EHMTI-0169. Trajectories of headache days over one year (5 waves) in chronic and episodic migraineurs participating in the chronic migraine epidemiology and outcomes (cameo) study. The Journal of Headache and Pain 2014 15(Suppl 1):D4. 